\title{
Characterization of Photonic Colloidal Single Crystals by Microradian X-ray Diffraction**
}

\author{
By Job H. J. Thijssen, Andrei V. Petukhov, Dannis C. 't Hart, Arnout Imhof, \\ Catharina H. M. van der Werf, Ruud E. I. Schropp, and Alfons van Blaaderen*
}

Photonic crystals (PCs) can provide unprecedented control over both the emission and the propagation of light, allowing important applications in, for example, infrared telecommunications. ${ }^{[1-3]}$ However, fabrication and characterization of PCs is challenging owing to the large refractive-index contrast that is needed to open up a photonic bandgap. ${ }^{[4]}$ Here we demonstrate that microradian X-ray diffraction can be used to characterize various (inverse) PCs with lattice spacings as large as $1.3 \mu \mathrm{m}$ at different stages of their fabrication. We have even fabricated non-close-packed (non-cp) PC structures by selfassembly of colloidal microspheres in an external electric field. Inverse PCs have been obtained by infiltration of the colloidal-crystal templates with amorphous silicon. The size of the colloidal particles allows us to characterize the internal $3 \mathrm{D}$ structure of these crystal templates in both real and reciprocal space.

PCs are structures in which the refractive index varies periodically in space on a length scale comparable to the wavelength of light. ${ }^{[3]}$ If the refractive-index contrast is large enough, PCs can have a photonic bandgap, which is the photonic analogue of the electronic bandgap in semiconductors. ${ }^{[5-7]} \mathrm{A}$ promising route towards relatively cheap PCs with many crys-

[*] Prof. A. van Blaaderen, J. H. J. Thijssen, D. C. 't Hart, Dr. A. Imhof Soft Condensed Matter, Debye Institute, Utrecht University Princetonplein 5, 3584 CC, Utrecht (The Netherlands) E-mail: A.vanBlaaderen@phys.uu.nl

Dr. A. V. Petukhov

Van't Hoff Laboratory, Debye Institute, Utrecht University Padualaan 8, 3508 TB, Utrecht (The Netherlands)

C. H. M. van der Werf, Prof. R. E. I. Schropp

Surfaces, Interfaces, and Devices, Debye Institute, Utrecht University

Princetonplein 5, 3584 CC, Utrecht (The Netherlands)

[***] We thank Jacob Hoogenboom for particle synthesis and Yu Ling Wu for useful discussions on UV polymerization. Furthermore, we would like to thank Igor Dolbnya, Florian Meneau, Abdellatif Moussaid, Erik-Jan de Jong, Dirk Detollenaere, Jeroen Jacobs, Federico Zontone, Henri Gleyzolle, Patrick Feder, Andrei Fluerasu, Marc Diot, Cyril Ponchut, Anatoly Snigirev, Anders Madsen, and Wim Bras for the excellent support they provided both before and during our $\mathrm{X}$-ray diffraction measurements. This research is/was supported by NanoNed, a national nanotechnology program coordinated by the Dutch Ministry of Economic Affairs. Finally, the European Synchrotron Radiation Facility, the "stichting voor Fundamenteel Onderzoek der Materie (FOM)", and the "Nederlandse organisatie voor Wetenschappelijk Onderzoek (NWO)" are acknowledged for financial support. tal layers is colloidal self-assembly. Monodisperse microspheres can self-assemble into 3D periodic arrangements, analogous to thermodynamic crystal phases. ${ }^{[8]}$ These colloidal crystals are then used as templates for infiltration with a high-index material, such as silicon, after which the original template is removed by a wet chemical etch. ${ }^{[9,10]}$

The multistep fabrication process can significantly affect the final 3D structure of the PC. Therefore, it is important to structurally characterize the PCs at every stage of their fabrication. The colloidal-crystal templates can be characterized in three dimensions using optical techniques, such as confocal microscopy, after refractive-index matching using a suitable liquid. ${ }^{[11,12]}$ However, index matching is not an option for (silicon) infiltrated PCs. Moreover, even for dry crystals, which can be index matched at visible wavelengths, it would still be very useful to have a $3 \mathrm{D}$ characterization technique that does not involve index matching. The infiltration of the crystal with a liquid and subsequent removal of this liquid might affect the 3D structure. Because of the relatively weak interaction of $\mathrm{X}$-rays with matter, X-ray scattering is an excellent tool to probe the internal structure of these PCs, as has been demonstrated for sub-micrometer lattice spacings. ${ }^{[13,14]}$ The challenge for X-rays stems from the dramatic difference between the X-ray wavelength (ca. $1 \AA$ ) and the colloid diameter (ca. $1 \mu \mathrm{m}$ ), leading to tiny diffraction angles of the order of $10^{-4}$ rad. However, it has recently been demonstrated that the required microradian angular resolution is attainable. ${ }^{[15,16]}$ Here, we demonstrate that microradian X-ray diffraction yields clear information on the 3D structure of PCs from the position and the height of the Bragg reflections.

Colloidal crystals with a non-cp, body-centered tetragonal (bct) lattice structure were fabricated by sedimentation of a colloidal dispersion of 1.1 or $1.4 \mu \mathrm{m}$ diameter silica spheres in an AC electric field perpendicular to gravity. ${ }^{[17,18]}$ Once a crystal was grown, the particles were immobilized using a polymerization process, allowing the electric field to be switched off without the crystal losing its then metastable bct structure $^{[18,19]}$ (see Experimental). Close-packed (cp) crystals of similar spheres were obtained by vertical controlled drying. ${ }^{[9,20]}$ The silica templates were characterized by confocal microscopy and scanning electron microscopy (SEM). Some samples were infiltrated with amorphous silicon by low-pressure chemical vapor deposition (LPCVD). ${ }^{[21]}$

Diffraction experiments were performed at the beamlines BM26B "DUBBLE" and ID10A "TROÏKA" of the Eur- 
opean Synchrotron Radiation Facility. The setup was aligned such that an image of the source was created at the detector screen, thus increasing the $q$-space resolution, which is necessary to measure Bragg reflections at very small angles. Furthermore, in most experiments, X-rays were allowed to propagate freely towards the sample, after which the transmitted and diffracted beams were focused by a compound refractive lens positioned just after the sample. ${ }^{[15,16]}$

We analyzed the diffraction data in terms of a hexagonal lattice. The reason for this is that all our crystals, as is often the case in colloidal self-assembly, consist of stackings of hexagonally packed layers, which orient parallel to the substrate. In bct crystals these layers are ABAB bridge-site stacked, yielding lines of particles in a real-space $z$-projection (see Fig. 1a). The conventional bct unit cell, the direction of the electric field $\boldsymbol{E}$, the laboratory $x y z$-frame, and the real-space lattice vectors $\boldsymbol{a}_{1}, \boldsymbol{a}_{2}, \boldsymbol{a}_{3}$ are given in Figure $1 \mathrm{~b}$. The cp structures, on the other hand, consist of hollow-site stacked layers with three possible lateral positions: A, B, and C (see Fig. 1c). The $2 \mathrm{D}$ reciprocal lattice of a single hexagonal plane is hexagonal and can be generated by two basis vectors, $\boldsymbol{b}_{1}$ and $\boldsymbol{b}_{2}$ (Fig. 1d). The 3D reciprocal lattice depends on the stacking of the layers. Any scattering vector can be decomposed into $\boldsymbol{q}=\boldsymbol{h} \boldsymbol{b}_{1}+k \boldsymbol{b}_{2}+\boldsymbol{l} \boldsymbol{b}_{3}$, where $\boldsymbol{b}_{3}$ is taken along the $z$-direction with a length $b_{3}=2 \pi / d$ determined by the interplanar spacing $d$. This non-primitive, reciprocal-space unit cell facilitates easy comparison between the scattering by $\mathrm{cp}$ and bct structures.

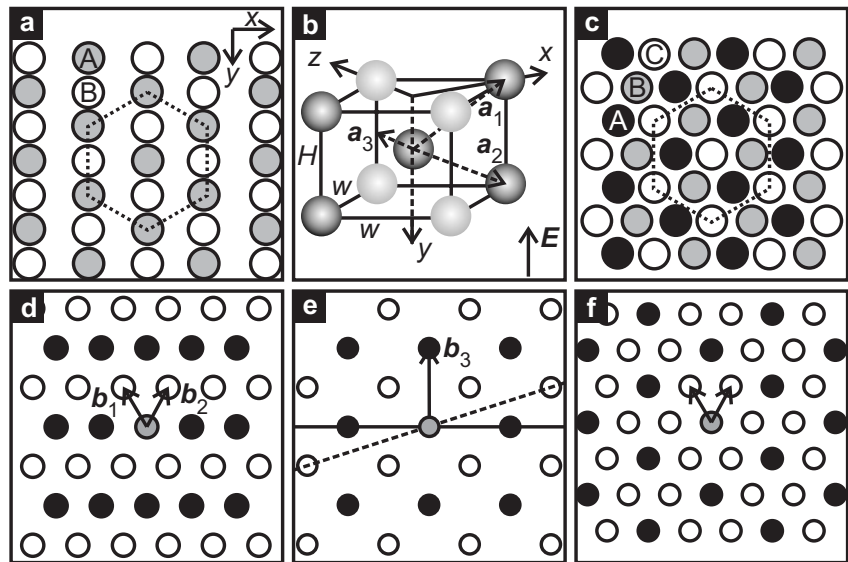

Figure 1. a) Projection of the bct structure along the z-axis, which is perpendicular to the hexagonal planes (see panel b), revealing bct $A B A B$ bridge-site stacking. b) Conventional unit cell for the bct real-space structure. The hexagonal plane $(x y)$ is indicated by the dark-gray spheres. The real-space lattice vectors are labeled $\boldsymbol{a}_{1}, \boldsymbol{a}_{2}$, and $\boldsymbol{a}_{3}$. c) Projection of an fcc (face-centered cubic) structure along the z-axis, revealing $A B C A B C$ hollow-site stacking. In panels a-c, the spheres have not been drawn to scale for clarity. d,e) Cross sections through the bct reciprocal lattice along the hexagonal $(x y)$ plane (d) and the $x z$-plane (e). The black dots correspond to integer $/$ and the white dots to half-integer $I$. The reciprocal lattice vectors are labeled $\boldsymbol{b}_{1}, \boldsymbol{b}_{2}$, and $\boldsymbol{b}_{3}$. Furthermore, the Ewald planes for an angle of incidence of $0^{\circ}$ (solid line) and $18.43^{\circ}$ (dashed line) are shown in (e). $f$ ) Hexagonal ( $x y)$ plane of the reciprocal lattice of a cp crystal. At normal incidence $(I=0)$, only the stacking-independent lattice points in reciprocal space (black dots) are probed.
Note that the third Miller index $l$ can take fractional values, ${ }^{[2]}$ whereas $h$ and $k$ still have to be integers. ${ }^{[23,24]}$

For any given crystal orientation, only lattice points in reciprocal space that are intersected by the Ewald sphere are probed. As our colloidal particles are typically $10^{4}$ times larger than the X-ray wavelength, the Ewald sphere ${ }^{[23,24]}$ is nearly flat and oriented perpendicular to the incoming X-ray beam. At normal incidence $(l=0)$, interference is constructive for bct structures if $(h+k)$ is even and destructive if $(h+k)$ is odd (see Fig. 1d). For odd $(h+k)$, the stacking-induced phase difference between the layers is compensated at half-integer values of $l$, which can be reached by specific sample rotations (see Fig. 1e). For a cp crystal at normal incidence $(l=0)$, interference is always constructive for $(h-k)$ divisible by three (see Fig. 1f).

Figure 2 a presents the normal-incidence $(l=0)$, X-ray diffraction pattern of a seven-layer bct crystal in a mixture of water and dimethylsulfoxide (DMSO). As expected, reflections for which $(h+k)$ is even are much stronger than those for which $(h+k)$ is odd (see Fig. 2c). It is convenient to compare reflections at the same distance from the direct beam. In that case, no form factor correction is necessary to compare peak intensities. Forbidden reflections are still slightly visible, since destructive interference is not complete in bct if the number of layers is odd. This becomes especially apparent if
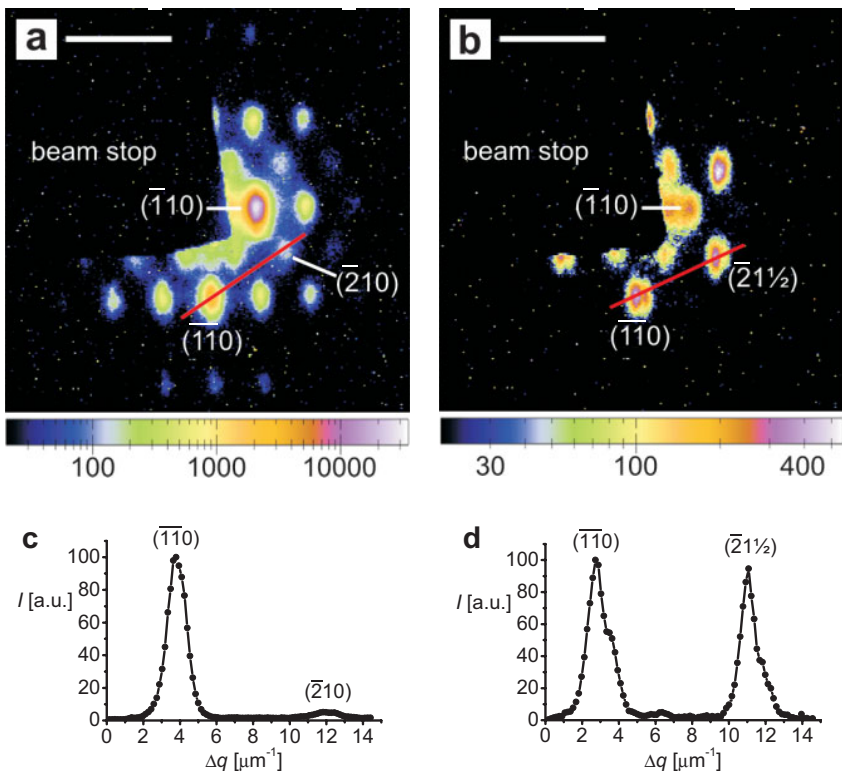

Figure 2. a) Diffraction pattern at normal incidence of a seven-layer bct crystal of $1.4 \mu \mathrm{m}$ diameter silica spheres in a water-DMSO mixture. b) Diffraction pattern of the same crystal at an angle of incidence of $18.44^{\circ}$. The $(\overline{1} 10)$ reflection has not disappeared, as it is on the axis of rotation ( $\gamma$-axis, see Fig. 1e). The white scale bar in both images is $10 \mu \mathrm{m}^{-1}$ The numbers below the intensity scale bar are detector pixel values. c,d) Line profiles through the (1)10) and (210) or (211/2) reflections, as in dicated in (a) and (b) by a red line. Both reflections are at the same dis tance from the direct beam, which means the value of the form factor is equal for both reflections. In both graphs, the maximum intensity $(I)$ of the highest peak was scaled to 100 . 
the number of layers is small. Note that the microradian resolution of the setup allows us to resolve even those Bragg reflections that are very close to the direct beam, such as the (110) reflection at a diffraction angle of only $72 \mu \mathrm{rad}$. To further clarify its structure, the bct crystal was rotated to vary the angle of incidence of the X-ray beam. Single-scattering theory predicts that the $(\overline{2} 1 l)$ reflection, which is forbidden for $l=0$, should show up if the sample is rotated over an angle of $18.43^{\circ}$ around the vertical $y$-axis. Indeed, the $\left(\overline{2} 1 \frac{1}{2}\right)$ reflection is observed and seen to be nearly as strong as the (1110) reflection (Fig. 2d). The ( $\overline{1} \overline{1} 0)$ reflection is still visible in Figure $2 b$ because it is on the axis of rotation (see Fig. 1e).

One of the major advantages of X-rays is that the internal $3 \mathrm{D}$ structure of samples with a large index contrast in the visible region can still be probed. Figure 3 a shows a normal-incidence $(l=0)$ X-ray diffraction pattern of an approximately six-layer cp crystal of silica spheres in air. The reflections for which $(h-k)$ is divisible by three, like the six reflections of the (110) family and the six reflections of the (220) family, are much stronger than the other reflections, as expected (see Fig. 1f). Note that $(h-k)$ is also divisible by three for the six reflections of the (300) family, but these appear close to the third minimum of the sphere form factor, reducing their intensity significantly. The appearance of forbidden reflections, like the six reflections of the (100) family, can be attributed to the finite number of layers and/or the possible stacking disorder. ${ }^{[25]}$ The refractive-index contrast can be enhanced even further by infiltration of the crystal with amorphous silicon. Figure $3 b$ shows a similar diffraction pattern of an approximately 20-layer cp crystal, which was partially infiltrated with amorphous silicon (estimated layer thickness $21 \mathrm{~nm}$ ). As in Figure $3 \mathrm{a}$, the dominant features are the six reflections of the (110) family and the six reflections of the (220) family (see Fig. 1f), demonstrating that cp crystals can be silicon-infiltrated by LPCVD without causing any significant damage.
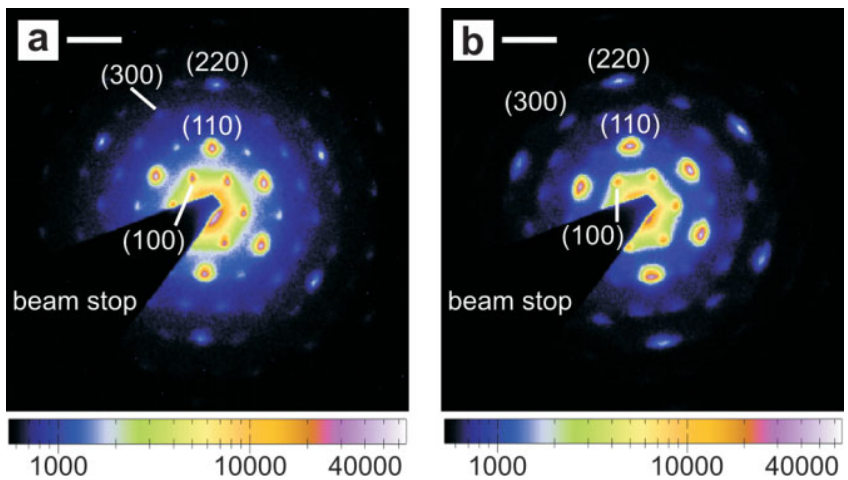

Figure 3. a) Diffraction pattern at normal incidence of an approximately six-layer cp crystal of $1.1 \mu \mathrm{m}$ diameter silica spheres in air. The stackingindependent reflections (see Fig. 1f) are much stronger than the other reflections. b) Normal-incidence diffraction pattern of a cp crystal with approximately 20 layers after partial infiltration with amorphous silicon. The white scale bar in both images is $10 \mu \mathrm{m}^{-1}$. The numbers below the intensity scale bar are detector pixel values.
The forbidden reflections are less pronounced than in Figure $3 \mathrm{a}$, which is due to the larger number of layers.

Finally, Figure 4 demonstrates that X-ray diffraction can be used to characterize even non-cp PCs at various stages of their fabrication. Figure 4a shows a normal-incidence X-ray diffraction pattern of an approximately 15-layer bct crystal of $1.4 \mu \mathrm{m}$ diameter silica spheres in a water-DMSO mixture. We can check that the structure is bct in real space by looking at a $z$-projection of a confocal 3D scan spanning six crystal layers, clearly revealing bct bridge-site stacking (see Fig. 4g). After solvent evaporation and subsequent refilling for index matching, confocal microscopy measurements did not indicate that the crystals suffer any serious damage upon solvent evaporation (see Fig. 4h). This was confirmed by X-ray measurements (see Fig. 4b), although the intensity of the forbidden reflection (120) seems slightly larger. The latter suggests that only a very small part of the crystal, if any at all, has a cp structure (see Figs. 1f and 3). Owing to the large capillary forces applied during solvent evaporation, slippage of a layer from the bridge sites to the hollow sites can cause part of a crystal to relax from bct to $\mathrm{cp}$.

Once the crystals have been infiltrated with amorphous silicon, it becomes impossible to characterize their 3D structure quantitatively in real space. Figure $4 \mathrm{i}$ shows an SEM image of an infiltrated bct crystal that was cleaved after infiltration. The space in between the spheres has been filled with silicon and the hexagonal layers are still ABAB stacked. The crystal seems to have taken quite some damage, but this might have been caused by the cleaving as well. One of the advantages of $\mathrm{X}$-ray diffraction is that it can probe the 3D structure in situ. Furthermore, it yields macroscopically averaged structural data, whereas SEM only provides information on a small part of the surface. Figure 4c shows the diffraction pattern of a similar, seven-layer crystal. Although the bct fingerprint is still visible in the diffraction pattern, the forbidden $(\overline{2} 10)$ reflection is nearly as strong as the allowed (1110) reflection, which means large parts of the crystal are no longer bridgesite but hollow-site stacked. The inset of Figure $4 \mathrm{i}$ indeed shows that the bottom two layers are hollow-site stacked, which is probably due to adhesion of the particles of the bottom layer to the glass substrate.

From a projection such as Figure $4 \mathrm{~g}$, we can determine in real space the ratio $a$ of the distance between the lines of particles and the distance between the particles in a line. This ratio $a$ can also be determined in reciprocal space by comparing distances along the (110) and (110) directions. For a perfect bct crystal with touching spheres, $\alpha$ would equal $\sqrt{3}$. From the $\mathrm{X}$-ray diffraction pattern shown in Figure 4a, a value for $a$ of $(1.023 \pm 0.002) \sqrt{3}$ can be extracted, whereas confocal microscopy on the same sample yields $(1.017 \pm 0.005) \sqrt{3}$. The values for $\alpha$ agree quite well and they both indicate that the hexagonal planes are compressed along the direction of the external electric field. ${ }^{[18]}$

We have demonstrated that X-ray scattering is an excellent probe of the internal 3D structure of $\mathrm{cp}$ and non-cp colloidal PCs with lattice spacings of the order of a micrometer. For ex- 


\section{ADYANCEP
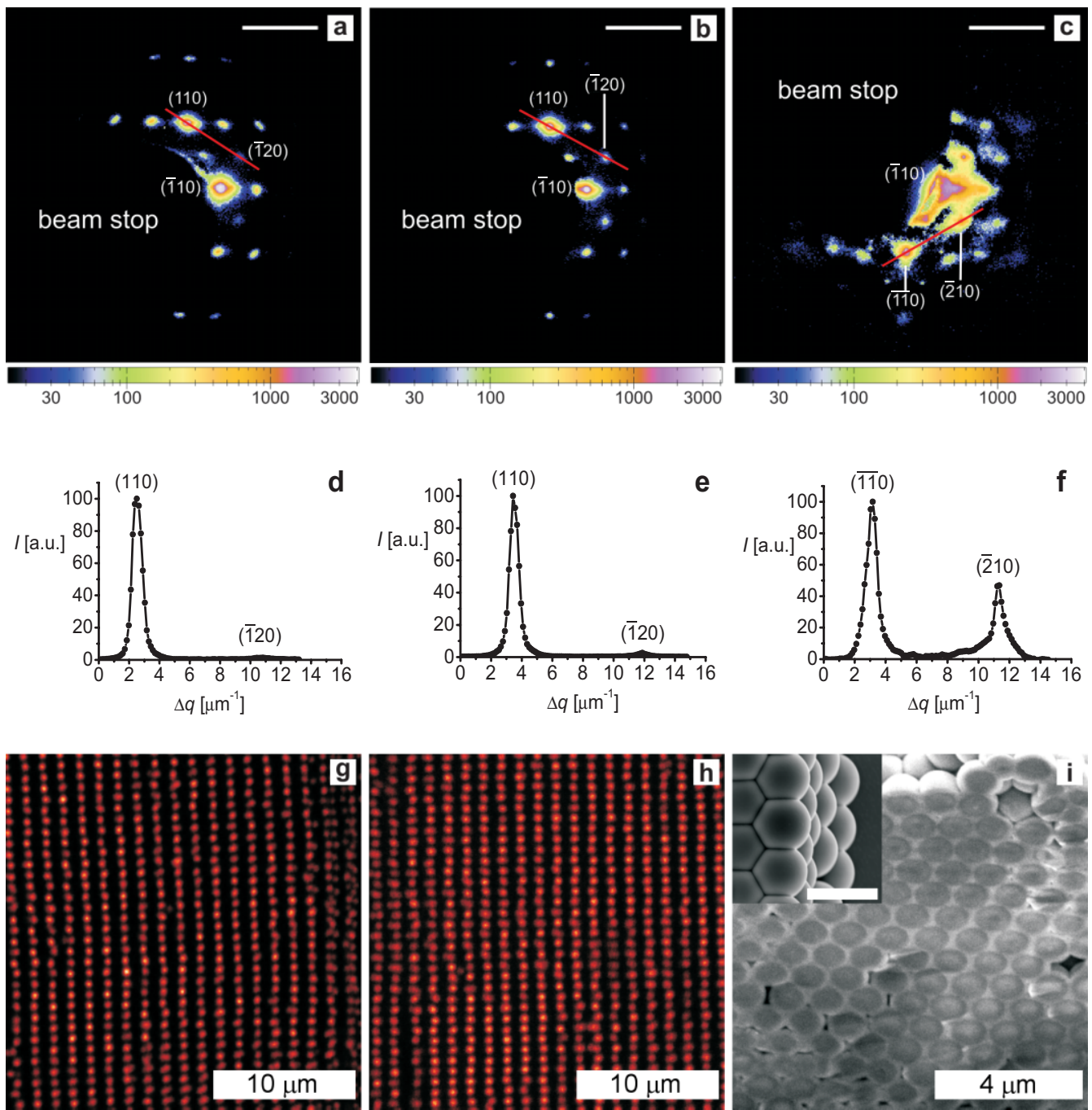

Figure 4. a-c) Normal-incidence X-ray diffraction patterns of a bct crystal consisting of $1.4 \mu \mathrm{m}$ diameter silica spheres; a) in a water-DMSO mixture, b) after solvent evaporation, and c) after infiltration with amorphous silicon. The number of layers is approximately 15, 15, and 7, respectively. The white scale bar in all images is $10 \mu \mathrm{m}^{-1}$. The numbers below the intensity scale bar are detector pixel values. The reflections in this figure appear to be much narrower than those in Figures 2,3. This is because an undulator source instead of a bending magnet source was used. $d-f$ ) Line profiles taken along the red lines in the diffraction images. g,h) Projections along the $z$-axis of 3D confocal microscopy scans spanning six hexagonal layers of a bct crystal of $1.4 \mu \mathrm{m}$ diameter silica spheres, both before (g) and after (h) solvent evaporation and refilling. i) SEM image showing the $y z$-plane, at a tilt angle of $45^{\circ}$, of a silicon-infiltrated bct crystal. The inset shows a top view $(x y)$ of a similar crystal at larger magnification. The scale bar in the inset is $2 \mu \mathrm{m}$.

ample, using this advanced scattering technique, we have found that the 3D structure of non-cp colloidal crystals can be significantly altered in going from dried to silicon-infiltrated crystals. Furthermore, for the first time, we have performed a comparison of both real-space and reciprocal-space 3D structural data of similar colloidal crystals. Up until now, we have only considered the position and the intensity of the diffraction peaks. The microradian resolution of our X-ray diffraction setup also allows accurate determination of the width of the Bragg reflections. Thus, important information on the presence of defects and long-range order can be obtained as well, even for samples that scatter strongly in the visible.

\section{Experimental}

Sample Preparation: All dispersions consisted of core/shell silica spheres [26-28] with a total diameter of 1.1 or $1.4 \mu \mathrm{m}$, as determined by static light scattering. Transmission electron microscopy measurements showed that the relative width of the size distributions was $2.5 \pm 0.5 \%$. The $400 \mathrm{~nm}$ diameter silica cores of all the particles were labeled with fluorescein isothiocyanate (FITC). The bct crystals were fabricated from dispersions of such spheres in a mixture of water and DMSO ( $87 \mathrm{vol} \%$ ) by sedimentation onto a glass cover slide (MenzelGläser, \#1 cover slip, ca. $150 \mu \mathrm{m}$ thick) in an AC electric field (ca. $70-140 \mathrm{~V} \mathrm{~mm}^{-1}, 1.000 \mathrm{MHz}$ ) perpendicular to gravity $[17,18]$. As the sample cell volume was fixed, the number of layers in these samples was determined by the volume fraction of silica spheres in the colloidal dispersion (e.g., $16.5 \mathrm{vol} \%$ yields ca. 15 layers). The parti- 
cles were immobilized by a polymerization process, which is the result of the interaction between the solvent and the glue that was used to seal the sample cells (Bison Epoxy Rapide) [18]. However, the sample used for Figure 4i was fabricated using a different polymerization process. In this case, the $1.1 \mu \mathrm{m}$ diameter silica particles were dispersed in a $10.1 \mathrm{vol} \%$ solution of trimethylolpropane ethoxylate triacrylate (Sigma-Aldrich, average molecular weight $=428 \mathrm{~g} \mathrm{~mol}^{-1}$ ) in DMSO (Merck, $>99.6 \%$ ). Next, $10 \mu \mathrm{L}$ of a $1.00 \mathrm{vol} \%$ solution of photoinitiator 2-hydroxy-2-methyl-propiophenone (Sigma-Aldrich, 97\%) in DMSO was added to $50 \mu \mathrm{L}$ of the dispersion. The resulting dispersion was used for bct crystal growth [18]. The particles in the crystal were subsequently immobilized by illumination with UV light (UVP, UVGL-58, $365 \mathrm{~nm}, 6 \mathrm{~W}$ ) for $2 \mathrm{~min}$ at a distance of approximately $1 \mathrm{~cm}$.

Infiltration of the colloidal crystals was performed using LPCVD [21]. Disilane $\left(\mathrm{Si}_{2} \mathrm{H}_{6}\right)$ was used as the precursor gas. In a typical LPCVD run, gas flows of $10 \mathrm{sccm}$ for the disilane gas and $100 \mathrm{sccm}$ for the hydrogen gas were used. The temperature at the sample was approximately $450{ }^{\circ} \mathrm{C}$, the pressure was $0.020 \mathrm{mbar}$, and the reaction duration was $300 \mathrm{~min}$. The LPCVD process results in the deposition of amorphous silicon, which has a refractive index that is slightly larger than that of crystalline silicon (3.59 instead of 3.5 [29]). Before infiltration, the bct crystals were heated up to at least $425^{\circ} \mathrm{C}$, at an average rate of $100{ }^{\circ} \mathrm{Ch}^{-1}$ at most. They were kept at the final temperature for at least $3 \mathrm{~h}$ in order to remove the polymer network, which was found to inhibit infiltration of the crystal layers below the top layer.

Sample Characterization: Confocal microscopy measurements were performed using a Leica TCS SP2 and TCS NT confocal scan head mounted on a Leica DM IRB inverted microscope. The crystal samples were index matched by infiltration with a mixture of water and DMSO (80 vol \% DMSO) or a mixture of water and glycerol (Merck, 87 vol \% glycerol). A Leica PL APO 100× (1.4 NA) oil-immersion objective was used, in combination with Cargille immersion oil (type B). The FITC was excited using the $488 \mathrm{~nm}$ line of an Ar laser. Dry and infiltrated crystals were (additionally) characterized using a Philips XL30FEG scanning electron microscope, operating at an accelerating voltage of $2-5 \mathrm{keV}$ and a working distance of approximately $5 \mathrm{~mm}$.

The synchrotron X-ray radiation was produced by either a bending magnet source ("DUBBLE") or an undulator source ("TROÏKA"). A typical photon energy of $11 \mathrm{keV}$ (wavelength $\lambda=1.1 \AA$ ) was selected using a $\mathrm{Si}(111)$ monochromator. At "DUBBLE", the diffraction patterns were recorded using a 16-bit charge-coupled device (CCD) camera (Photonic Science, Xios II) with a pixel size of $22.7 \mu \mathrm{m} \times 22.7 \mu \mathrm{m}$ and an image size of $1270 \times 1160$ pixels. The $q$-space calibration of the photonic science camera was performed at "DUBBLE" using dry rat-tail collagen, yielding an inherent uncertainty in absolute $q$-space distances of $3 \%$. At "TROÏKA", a 12-bit CCD camera (Sensicam, PCO CCD Imaging) was used, with a pixel size of $6.7 \times \mu \mathrm{m} 6.7 \mu \mathrm{m}$ and an image size of $1280 \times 1024$ pixels. The Sensicam camera was calibrated using a cp reference crystal, which had been measured at "DUBBLE" as well. Because of the limited dynamic range of $\mathrm{CCD}$ cameras, diffraction patterns were recorded several times with exposure times ranging from $1 \mathrm{~s}$ up to $10 \mathrm{~min}$. The variation in exposure time and sample absorption complicates the comparison of the intensities of corresponding reflections in different diffraction patterns presented here. All diffraction patterns have been corrected for background scattering. The peak profiles in Figures 2 and 4 were obtained by averaging the intensity over a band of five to seven detector pixels wide. Distances in reciprocal space were measured between the maxima of allowed Bragg reflections, whose positions were determined by fitting Gaussian curves to their line profiles.

Received: December 23, 2005

Final version: April 3, 2006

[1] P. Lodahl, A. F. van Driel, I. S. Nikolaev, A. Irman, K. Overgaag, D. Vanmaekelbergh, W. L. Vos, Nature 2004, 430, 654

[2] C. M. Soukoulis, Photonic Crystals and Light Localization in the 21st Century (Ed: C. M. Soukoulis), Kluwer, Dordrecht, The Netherlands 2001.

[3] J. D. Joannopoulos, R. D. Meade, J. N. Winn, Photonic CrystalsMolding the Flow of Light, Princeton University Press, Princeton, NJ 1995.

[4] H. S. Sözüer, J. W. Haus, R. Inguva, Phys. Rev. B 1992, 45, 13962.

[5] V. P. Bykov, Sov. J. Quantum Electron. 1972, 35, 269

[6] E. Yablonovitch, Phys. Rev. Lett. 1987, 58, 2059.

[7] S. John, Phys. Rev. Lett. 1987, 58, 2486

[8] P. N. Pusey, W. van Megen, Nature 1986, 320, 340.

[9] Y. A. Vlasov, X. Z. Bo, J. C. Sturm, D. J. Norris, Nature 2001, 414, 289.

[10] A. Blanco, E. Chomski, S. Grabtchak, M. Ibisate, S. John, S. W. Leonard, C. Lopez, F. Meseguer, H. Miguez, J. P. Mondia, G. A. Ozin, O. Toader, H. M. van Driel, Nature 2000, 405, 437.

[11] A. van Blaaderen, P. Wiltzius, Science 1995, 270, 1177.

[12] A. van Blaaderen, R. Ruel, P. Wiltzius, Nature 1997, 385, 321.

[13] M. Megens, C. M. van Kats, P. Bösecke, W. L. Vos, J. Appl. Crystallogr. 1997, 30, 637.

[14] W. L. Vos, M. Megens, C. M. van Kats, P. Bösecke, Langmuir 1997, 13,6004 .

[15] M. Drakopoulos, A. Snigirev, I. Snigireva, J. Schilling, Appl. Phys. Lett. 2005, 86, 014102.

[16] A. V. Petukhov, J. H. J. Thijssen, D. C. 't Hart, A. Imhof, A. van Blaaderen, I. P. Dolbnya, A. Snigireva, A. Moussaïd, A. Snigirev, J. Appl. Crystallogr. 2006, 39, 137.

[17] A. Yethiraj, A. van Blaaderen, Nature 2003, 421, 513.

[18] A. Yethiraj, J. H. J. Thijssen, A. Wouterse, A. van Blaaderen, Adv. Mater. 2004, 16, 596.

[19] P. Jiang, M. J. McFarland, J. Am. Chem. Soc. 2004, 126, 13778

[20] P. Jiang, J. F. Bertone, K. S. Hwang, V. L. Colvin, Chem. Mater. 1999, $11,2132$.

[21] A. Madan, P. Rava, R. E. I. Schropp, B. von Roedern, Appl. Surf. Sci. 1993, 70/71, 716 .

[22] W. Loose, B. J. Ackerson, J. Chem. Phys. 1994, 101, 7211.

[23] J. M. Ziman, Principles of the Theory of Solids, Cambridge University Press, New York 1995.

[24] A. Guinier, X-ray Diffraction: In Crystals, Imperfect Crystals, and Amorphous Bodies, Dover Publications, New York 1994.

[25] A. V. Petukhov, I. P. Dolbnya, D. G. A. L. Aarts, G. J. Vroege, H. N. W. Lekkerkerker, Phys. Rev. Lett. 2003, 90, 028304.

[26] W. Stöber, A. Fink, E. Bohn, J. Colloid Interface Sci. 1968, 26, 62.

[27] H. Giesche, J. Eur. Ceram. Soc. 1994, 14, 205.

[28] A. van Blaaderen, A. Vrij, Langmuir 1992, 8, 2921.

[29] J. Kalkman, E. de Bres, A. Polman, Y. Jun, D. J. Norris, D. C. 't Hart, J. P. Hoogenboom, A. van Blaaderen, J. Appl. Phys. 2004, 95, 2297. 